\title{
Photo-recall cutaneous reaction to gemcitabine
}

\author{
Simone D'Adamio *, Marina Talamonti, Luca Bianchi, Marco Galluzzo \\ Department of Dermatology, Policlinico Tor Vergata, University of Rome "Tor Vergata", Rome, Italy
}

\begin{abstract}
Cutaneous reactions attributable to chemotherapy too often result in a modification of patients' treatment plan. Most treatments remain largely unproven: only small series or case reports may indicate possible treatment options. Main observations: We reported the case of a male patient with gemcitabine-induced skin reaction occurred after a cycle of therapy with carboplatin plus gemcitabine following a 21 days' schedule. The patient came to our attention for an extensive, well-demarked, erythematous, lilaceous, warm indurated lesion, covering his dorsal faces of both hands, without systemic symptoms. Clobetasol propionate $0.05 \%$ ointment was prescribed as main therapy, followed by a cream containing boswellic acids. Conclusions: It is presumable that similar dermatological lesions consist in a 'radiation recall reaction' whereby an inflammatory reaction occurs in the area previously treated with radiotherapy or affected by a sun-burn in the past. In our patient, interestingly, there is no history of radiotherapy even if there is history of strong sun exposure. Pharmacological antiinflammatory effect due to boswellic acids was studied and relieved only in radiation-induced dermatitis, and even if larger studies would have been set in order to have more effective results, it would be useful to study application of this compound in chemotherapy-induced cutaneous adverse reactions too.
\end{abstract}

Keywords: gemcitabine, chemotherapy-related skin toxicity, photo-recall skin reaction, boswelic acids

\section{Introduction}

Over the past several decades, great advances have been accomplished in the development of chemotherapeutic and molecularly targeted agents that greatly improved survival and quality of life for people suffering for cancer [1].

The growing international cancer burden, combined with increased availability of newer agents, will undoubtedly lead to an increase in the incidence of chemotherapy-related skin toxicity. Cutaneous reactions attributable to chemotherapy too often result in a modification of patients' treatment plan. Small series or case reports may indicate possible treatment options, but most remain largely unproven. Certain toxicities can be effectively treated or prevented with opportune premedication (for

Received: October 2017; Accepted after review: December 2017; Published: December 2017.

${ }^{*}$ Corresponding author: Simone D'Adamio, University of Rome "Tor Vergata", Department of Dermatology, Policlinico Tor Vergata, Viale Oxford 81, 00133 Rome, Italy

Email: simonedadamio1988@tiscali.it example, prophylactic mouthwashes to prevent mucositis), otherwise most of the toxicities are only reversible with chemotherapy dose reductions or, in extremis ratio, with delays $[1,2]$.

Gemcitabine (2,2-difluorodeoxycytidine) is used in the treatment of many solid cancer types including breast, endometrium, pancreas, bladder, ovarian, lung (non-small cell), and colon cancers and malignant mesothelioma.

Gemcitabine and carboplatin combination chemotherapy seems to be superior to gemcitabine single therapy in terms of response rate and overall survival with comparable non-hematologic toxicities. Its toxic effects are myelosuppression, nausea and vomiting, flu-like syndrome, altered liver function tests, proteinuria, hematuria, bronchospasm, somnolence, alopecia, rash, itching, and fever [3-5].

We reported the case of a male patient with gemcitabine-induced cellulitis-like skin reaction occurred after a cycle of therapy with carboplatin plus gemcitabine following a 21 days' schedule. We tried to focus on this 
adverse reaction, considering that few similar cases are reported in people treated with gemcitabine $[5,6]$.

\section{Case Report}

An 86 male patient came to our attention, sent by oncology department as consultancy. The patient was started to a combined chemotherapy after the diagnosis of metastatic lung cancer. His therapy schedule was: gemcitabine $1000 \mathrm{mg} / \mathrm{m} 2$ at day 1 and 8; carboplatin AUC 5 at day 8. The cycle had to be repeated every 21 days.

The clinical examination of the skin revealed an extensive, well-demarked, erythematous, violaceous, warm indurated lesion, covering the dorsal faces of both hands (Figure 1a).

The patient did not suffer for fever, or other systemic symptoms, but the complaints included local functional impairment, and burning and stinging sensation. The patient had no history of previous radiotherapy. The lesions, only localized on the hands, started to set after the second infusion of gemcitabine, on the eight day of the first cycle. The oncology team decided to postpone the second chemotherapy session until the complete remission of the lesions.

Considering the possibility of a gemcitabine adverse reaction, we prescribed to the patient a corticosteroid based local therapy. Clobetasol propionate $0.05 \%$ ointment was prescribed in occlusion for 7 days bis in die. As maintenance therapy, we prescribed a base cream, containing boswellic acids in a proprietary formulation (Bosexi $(\mathbb{B})$, palmitoiletanolamide (PEA) and Extremosio ${ }^{\circledR}$, a liposomal component containing trealose. Furthermore, we prescribed solar protection, in order to avoid photosensitivity reactions, considering that the patients usually stay outside during the day for recreational reasons (he lives in countryside). We did not prescribe antibiotics, according to the relative frequency of non-infectious adverse reactions in people treated with gemcitabine $[5,6,8,9]$.

We evaluated the patient after a week, and then after two weeks. After seven days, swelling was highly reduced, and lesion started to be scaling. Erithema was improving, and the same was for burning and stinging sensation. After two week, patient showed only residual depigmentation. All symptoms were resolved and functional impairment disappeared (Figure 1b).

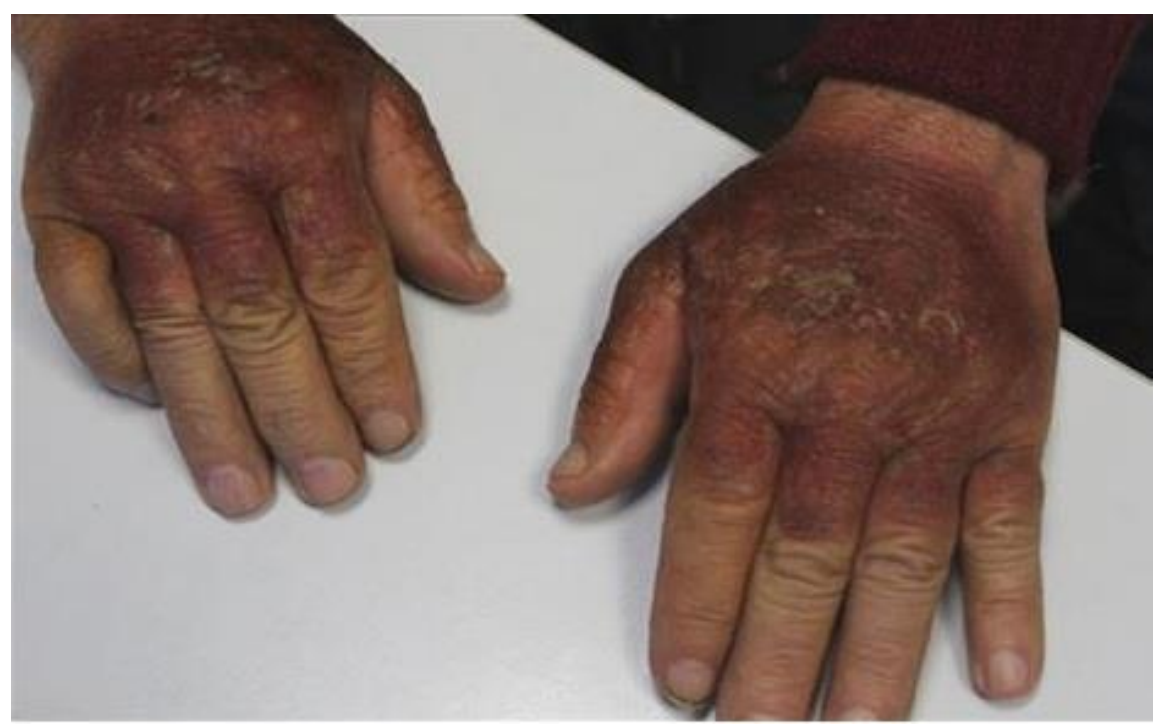

Fig. 1a. Well-demarked, erythematous, violaceous, warm indurated lesion, covering his dorsal faces of both hands after an entire cycle of chemotherapy. 


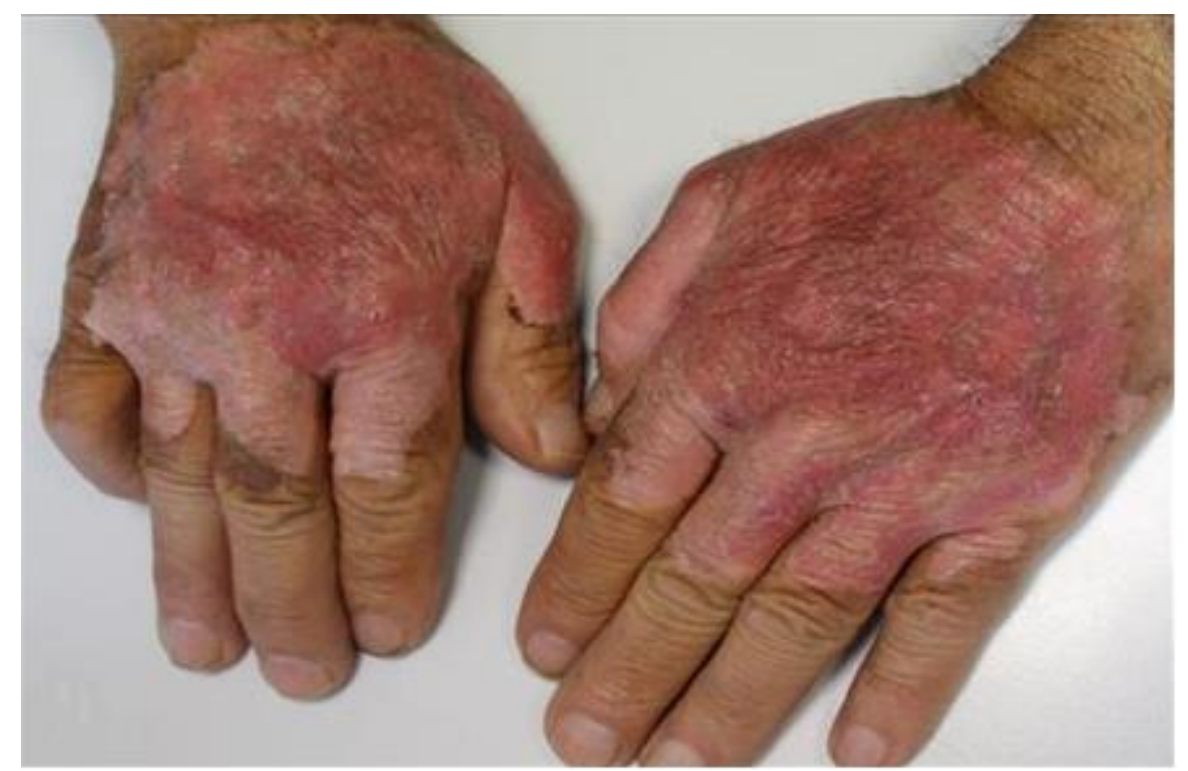

Fig. 1b. Skin aspect after two weeks with: clobetasol ointment applied as first-line therapy for a week, plus a base cream containing boswellic acid as maintenance therapy.

According to the improvement of patient's condition, the oncologists' decision was to start the second cycle of treatment.

During the second cycle of treatment, patient continued to apply the prescribed maintenance therapy with no more cutaneous adverse manifestations. The patient did not experience any recurrence in lesions during the whole chemotherapy. No other adverse reactions were reported.

\section{Discussions}

There are many cutaneous reactions resulting from use of various chemotherapeutics. It is important to recognize these adverse effects and differentiate them from infectious or other causes, in order to provide logical and compassionate care. Despite the frequency of cutaneous toxicities with chemotherapeutic and molecularly targeted agents used in cancer therapy, little is understood about the pathogenesis of the reactions [1].

It is highly suspected that, in our case, skin symptoms are attributable to gemcitabine or its metabolites. It is presumable that these lesions involved with gemcitabine consist in a 'radiation recall reaction', or alternatively a UVrecall reaction, whereby an inflammatory reaction occurs in the area previously treated with radiotherapy or involved in a sunburn in the past. Actual mechanism of this is not fully understood. Maybe, recovering keratinocytes being damaged by the chemotherapy drugs play a key role in these lesions, thus these cells are the ones which divide and regenerate most rapidly. Our patient, however, had no history of previous radiotherapy. While the 'radiation recall phenomenon' induced by some cytotoxic drugs, especially gemcitabine, is well known, to our knowledge only few cases of gemcitabine related toxicity in not previously irradiated skin have been reported. In our case, we must underline that the patient is a person with a strong history of sun exposure. Some authors explicate similar cutaneous reactions, arguing that in areas with impaired lymphatic drainage, such as lower limbs, gemcitabine pharmacokinetics might be altered. Actually, inactivation happens slower, and the drug might accumulate in the subcutaneous and cutaneous tissue, contributing to local toxicity. [7-12]

Extracts of the resin of Boswellia serrata (B. serrata) exert anti-inflammatory and antiphlogistic activities. Anti-inflammatory effect of Boswellic acids (BAs) was studied and relieved in radiation-induced dermatitis. Even if larger studies would have been set, in order to have more effective results, it would be useful to study application of this compound in 
chemotherapy-induced cutaneous adverse reactions too [7].

Therapy for the cutaneous toxicities of chemotherapeutic agents, in fact, remains largely supportive, with withdrawal of the offending agent as the most effective means of alleviating toxicity. Small series or case reports may indicate possible treatment options, but most remain largely unproven [1].

\section{Conclusions}

It is presumable that similar dermatological lesions consist in a 'radiation recall reaction' whereby an inflammatory reaction occurs in

\section{References}

1. Sanborn RE, Sauer DA. Cutaneous reactions to chemotherapy: commonly seen, less described, little understood. Dermatol Clin 2008; 26:103119.

2. Wyatt AJ, Leonard GD, Sachs DL. Cutaneous reactions to chemotherapy and their management. Am J Clin Dermatol 2006; 7(1):45-63.

3. Sederholm C, Hillerdal G, Lamberg K. Phase III trial of gemcitabine plus carboplatin versus single-agent gemcitabine in the treatment of locally advanced or metastatic non-small-cell lung cancer: the Swedish Lung Cancer Study Group. J Clin Oncol 2005; 23(33):8380-8388.

4. Yuh YJ, Lee HR, Kim SR. Gemcitabine and carboplatin combination chemotherapy for elderly patients with advanced non-small cell lung cancer: a feasibility study. Cancer Res Treat 2008; 40(3):116-120.

5. Kuku I, Kaya E, Sevinc A, et al. Gemcitabineinduced erysipeloid skin lesions in a patient with malignant mesothelioma $J$ Eur Acad Dermatol Venereol 2002; 16:271-272.

6. Tan DH, Bunce PE, Liles WC, et al. Gemcitabine-related "pseudocellulitis": report of 2 cases and review of the literature. Clin Infect Dis 2007; 45:e72-76. the area previously treated with radiotherapy or affected by a sun-burn in the past. In our patient, interestingly, there is no history of radiotherapy even if there is history of strong sun exposure. Pharmacological antiinflammatory effect due to boswellic acids was studied and relieved only in radiation-induced dermatitis, and even if larger studies would have been set in order to have more effective results, it would be useful to study application of this compound in chemotherapy-induced cutaneous adverse reactions too.

\section{Conflict of interest}

The authors declare no conflict of interest.

7. Togni S, Maramaldi G, Bonetta A, et al. Clinical evaluation of safety and efficacy of Boswellia based cream for prevention of adjuvant radiotherapy skin damage in mammary carcinoma: a randomized placebo controlled trial. Eur Rev Med Pharmacol Sci 2015; 19(8):1338-1344.

8. Brandes A, Reichmann U, Plasswilm L, et al. Time- and dose-limiting erysipeloid rash confined to areas of lymphedema following treatment with gemcitabine - a report of three cases. Anticancer Drugs 2000; 11(1):15-17.

9. Korniyenko A, Lozada J, Ranade A, et al. Recurrent lower extremity pseudocellulitis. Am J Ther 2012; 19(4):e141-142.

10. Zustovich F, Pavei P, Cartei G. Erysipeloid skin toxicity induced by gemcitabine. J Eur Acad Dermatol Venereol 2006; 20(6):757-758.

11. Ruiz-Casado A, Gutiérrez D, Juez I. Erysipeloid rash: A rare adverse event induced by gemcitabine. J Cancer Res Ther 2015; 11(4):1024.

12. Tan E: Skin toxicity of chemotherapy drugs [https://www.dermnetnz.org/topics/skin-toxicityof-chemotherapy-drugs/\#4 available at 12.26.2017]. 